\title{
Uso de Emulsiones Pickering en la Reaccion de Obtención de Terpineol a partir de Aceite Esencial de Pomelo
}

\section{Use of pickering emulsions in the reaction of obtaining terpineol from grapefruit essential oil}

\section{Liliana M. Cáceres}

Centro de Investigación en Química Orgánica Biológica (FRRe, UTN)-Instituto de Modelado e Innovación Tecnológica (CONICET-UNNE). Resistencia, Chaco - Argentina Imarielc@yahoo.com.ar

\section{Silvia N. Zambón}

Centro de Investigación en Química Orgánica Biológica (FRRe, UTN)-Instituto de Modelado e Innovación Tecnológica (CONICET-UNNE). Resistencia, Chaco - Argentina silzambon@hotmail.com

\section{Gustavo A. Velasco}

Centro de Investigación en Química Orgánica Biológica (FRRe, UTN)-Instituto de Modelado e Innovación Tecnológica (CONICET-UNNE). Resistencia, Chaco - Argentina gavelasco@arnet.com.ar

\section{Ester R. Chamorro}

Centro de Investigación en Química Orgánica Biológica (FRRe, UTN)-Instituto de Modelado e Innovación Tecnológica (CONICET-UNNE). Resistencia, Chaco - Argentina mandhy@hotmail.com 


\title{
Resumen
}

El objetivo de este trabajo fue aumentar el rendimiento y selectividad en la reacción de obtención de a-terpineol a partir de limoneno, componente mayoritario del aceite esencial de pomelo, con el uso de emulsiones pickering de agua:aceite. Se utilizaron carbón activado, bentonita, sílica y alúmina como sólidos estabilizantes de la emulsión. Se caracterizaron las emulsiones midiendo su conductividad en distintas relaciones agua:aceite para determinar el punto donde ocurre la inversión de fase de la emulsión. Se prepararon las emulsiones pickering de tipo aceite/agua (o/w), estableciendo las concentraciones másicas óptimas de cada sólido. El rendimiento máximo obtenido en a-terpineol fue de un $43 \%$ utilizando sílica, un $36 \%$ más que en medio de reacción sin sólidos. También se logró reutilizar los sólidos dos veces sin diferencias en el rendimiento de la reacción.

Palabras clave: pickering, emulsión, a-terpineol, limoneno.

\begin{abstract}
The aim of this work was to increase the yield and selectivity in the reaction of obtaining a-terpineol from limonene, a major component of the grapefruit essential oil, with the use of water:oil pickering emulsions. The stabilizing solids of the emulsion were activated carbon, bentonite, silica and alumina. The emulsions were characterized by measuring their conductivity in different water:oil ratios to determine the point where the phase inversion of the emulsion occurs. The oil/water $(\mathrm{o} / \mathrm{w})$ type pickering emulsions were prepared, establishing the optimum mass concentrations of each solid. The maximum yield obtained in a-terpineol was $43 \%$ using silica, $36 \%$ more than in reaction medium without solids. It was also possible to reuse the solids twice without differences in the yield of the reaction.
\end{abstract}

Keywords: pickering, emulsion, a-terpineol, limonene.

\section{Introducción}

El terpineol es un alcohol monoterpénico, muy utilizado en la industria. Este compuesto tiene un aroma agradable similar a la lila, por ello se lo emplea en cosmética, perfumería y en la elaboración de limpiadores y detergentes. El consumo del terpineol se estima en más de 1.000 toneladas por año (Bathia y col., 2008: S275-S279) y es considerado uno de los 30 compuestos más usados como saborizantes (Welsh y col., 1989:105-169) en la industria alimentaria. La importancia de la producción del terpineol se debe también a sus propiedades antimicrobianas (Oyedemi y col., 2009:1280-1286). Generalmente el terpineol es obtenido a partir de la hidratación del a-pineno, o en menor grado del limoneno, ambos monoterpenos componentes de aceites esenciales muy utilizados como sustratos para la producción de sabores y fragancias (Gusevskaya, 2014:1506-1515; Rottava y col., 2010:1128-1131, Baptistella, y col., 2009:1069-1071). Estas reacciones utilizan catalizadores sólidos como zeolitas beta, modificadas con cationes metálicos por intercambio iónico (Yadav y col., 2009:101-109), materiales laminares tipo cobalto-molibdato (Salamanca y col., 2009:93-100), y heteropoliácidos (Avila y col., 2008:1460-1462; Robles-Dutenhefner y col., 2001:33-42). En la actualidad también se están utilizando catalizadores biológicos como Aspergillus niger (Castellanos Molina, 2007:80-82), Penicillium sp. 2025, Aspergillus sp. 2038, Fusarium oxysporum 152B 
(Maróstica y Pastore, 2007:345-350) para la obtención de terpineol por bioconversión del limoneno (Tan y col., 1998:29-31).

Las reacciones catalíticas que se llevan a cabo en sistemas heterogéneos están formadas por una mezcla bifásica, constituidas por dos sustancias inmiscibles como por ejemplo el agua y un líquido orgánico hidrófobo (Starks, 1971:195-199). En el caso de la obtención de terpineol, una fase está compuesta por el agua, y la otra parte (hidrófoba) por aceite esencial de pomelo, rico en limoneno. El rendimiento de esta reacción se ve afectado por la dificultad de la transferencia de masa entre ambas fases. Una forma de solucionar este problema es a través de la formación de una emulsión, cuyo objetivo es aumentar el área superficial de la interfase y por ende, la posibilidad de transferencia de materia (Schramm, 2005:10-12; Ramírez Galilea, 2012:8-12). Es importante señalar que estas emulsiones son termodinámicamente inestables y su formación no ocurre de manera espontánea, sino que requiere de distintas acciones como ser: un mezclado mecánico para unir ambas fases, la adición de un surfactante para disminuir su tensión interfacial (Salager y col., 1982:279-292) o la adición de partículas sólidas finas (Aranberri, 2009:211-231). Hace más de cien años se descubrió que las partículas sólidas generan una película resistente en la interface, entre las dos fases no miscibles, inhibiendo la coalescencia de las gotas de la emulsión, las que posteriormente fueron llamadas emulsiones pickering (Popp y col., 2010:39-58).

Por otra parte, una emulsión se puede clasificar de dos formas, de acuerdo a cuál sea la fase dispersa. Una emulsión de aceite en agua (o/w) se forma cuando las gotas de aceite constituyen la fase dispersa repartidas dentro del agua, y una emulsión de agua en aceite (w/o) se compone de gotas de agua repartidas en el aceite (Gers-Barlag y Müller, 2007; Varka y col., 2010:181-188; Monfort, 2004:147-148). Una partícula sólida puede estabilizar cualquiera de estas emulsiones convirtiéndolas en emulsiones pickering. Generalmente las partículas sólidas hidrofílicas tienden a formar emulsiones o/w, mientras que las partículas hidrofóbicas forman emulsiones w/o (Aveyard y col., 2003:503-546). El uso de estas partículas en reacciones con líquidos inmiscibles puede presentar una gran ventaja con respecto a los agentes surfactantes, ya que resultan significativamente más fáciles de separar de la mezcla bifásica una vez que finaliza la reacción (Aranberry y col., 2006:13-17). Hay varios estudios que demuestran que la naturaleza de la emulsión agua en aceite o viceversa depende no solo del tipo de partícula sino también de la proporción fase acuosa-fase orgánica (Aranberri, 2009:13-17; Piriyaprasarth y col., 2016:589-598). Además, (Wen y col., 2014:695700) encontraron que la estabilidad de las emulsiones depende del $\mathrm{pH}$ y de la temperatura. Ellos estudiaron una emulsión pickering de d-limoneno en agua estabilizada con nanocristales de celulosa, homogeneizada por dispersión ultrasónica. Otra propiedad físico-química que interesa de las emulsiones pickering es su conductividad. Debido a la naturaleza dispersa de las emulsiones, la fase continua es la que va a ser capaz de transportar carga eléctrica, y la fase dispersa no. Por tanto, una emulsión con fase externa acuosa (con presencia de electrolitos) va a tener una alta conductividad, mientras que una emulsión con fase externa orgánica va a tener una conductividad muy baja. Esta característica permite identificar el tipo de emulsión, ya sea o/w, w/o o inclusive múltiple (Salager y col., 1991:5967). Asimismo, un cambio brusco en la conductividad determina un cambio de fase en la emulsión, es decir, un cambio de un tipo de emulsión en otra (Salager y col., 1982:279-292; Piorkowski y Mc Clements, 2014:5-41). Es bien conocido el uso de microemulsiones, con superficies interfaciales extremadamente elevadas para facilitar algunos procesos químicos concretos. Esto aumenta las velocidades de reacción, y puede conducir a una formación más selectiva del producto (Newton y col., 2015:1243-1249; Garti, 2003:197-211; Yaghmur y 
col., 2005:223-234) con muchas aplicaciones en la industria, como catálisis (Laguna y col., 2016:140-149), farmacia (Xinga y col., 2016:709-718), combustibles (Pereira y col., 2016:6064), alimentos (Garti, 2003:197-211; De Campo y col., 2004:251-267) y otros (Kasaka y col., 2016:49-58; Piorkowski y Mc Clements, 2014:5-41). En una emulsión pickering, la reacción se produce por el uso de pequeñas cantidades de un agente sólido que transfiere un reactante a través de la interfaz a la otra fase. De este modo, las reacciones entre dos sustancias pueden proceder por aumento del contacto entre ellas. El agente no se consume, sino que mantiene la función de transporte (Starks, 1971:195-199). Estas partículas sólidas pueden ser orgánicas (Hu y col., 2016:300-310), inorgánicas (Yang y col., 2017:213-222) u otras (Low y col., 2017:391-399; Zhang y col., 2009:110-116). Recientemente las emulsiones pickering han sido de mucho interés debido a su gran campo de aplicación, por ello se requiere el desarrollo de nuevas e inteligentes emulsiones de este tipo con estabilidad controlable que satisfaga la demanda en aplicaciones constantemente actualizadas (Yang y col., 2017:213-222).

En este trabajo se reporta la utilización de partículas sólidas como bentonita, alúmina, sílice y carbón activado para la formación de emulsiones pickering con aceite esencial de pomelo y agua con el fin de obtener a-terpineol.

\section{Desarrollo}

\section{Materiales}

El aceite esencial de pomelo (Citrus paradisi) fue obtenido como subproducto en una planta industrial de extracción de zumo de cítricos de la ciudad de Bella Vista, provincia de Corrientes, Argentina. El Índice de refracción (según Norma IRAM-SAIPA 18505:2002) fue de 1,4723; La Densidad relativa (según Norma IRAM-SAIPA 18504:2002) de 0,8573; y la rotación óptica (según Norma IRAM-SAIPA 18507:1987) de 91,0. Los componentes identificados mediante cromatografía de gases acoplada a espectrometría de masas (GC/MS) representan el 99,10\% de los componentes del aceite esencial de pomelo. El limoneno, fue el monoterpeno identificado en mayor proporción, (92,60\%); otros monoterpenos encontrados fueron $\beta$-mirceno (1,2\%) y a-pineno (0,6\%) (Cáceres y col., 2018, 31:30-37; Vasek y col., 2015:16-26). Se utilizó: Agua deionizada, $0,06 \mathrm{mScm}^{-1}$ de conductividad y ácido tricloroacético (Fluka, p.a.); y como estabilizantes sólidos, carbón activado (AEB group), sílica (Fluka), alúmina (Sigma Aldrich), bentonita (Biopack).

\section{Formulación y caracterización de las emulsiones}

Primeramente, se prepararon las emulsiones de agua con $0,06 \mathrm{mScm}^{-1}$ de conductividad y aceite esencial de pomelo. El ensayo inicial se realizó con de $0,2 \mathrm{~g}$ de agua y $1 \mathrm{~g}$ de aceite. Se prepararon distintas emulsiones aumentando la masa de agua hasta llegar a 4 g. Se agitó a $1500 \mathrm{rpm}$ durante 5 minutos. Por otra parte, se prepararon 4 grupos de emulsiones pickering agua-aceite esencial con carbón activado, bentonita, sílice y alúmina. Todos los grupos se formularon con las mismas fracciones aceite:agua que las emulsiones sin sólido. La cantidad de sólido a agregar a cada grupo se determinó probando distintas masas del mismo agitando hasta llegar a una emulsión homogénea y estable (aquella que una vez suspendida la agitación no se separa en dos fases transcurrida una hora). Se midió la conductividad de todas las emulsiones con un conductímetro Oakton-con 510 Eutech Instruments, Thermo Fisher Scientific, para luego obtener el punto de inversión del tipo de emulsión. Además, se realizó la prueba de la gota en cada una, dejando caer gotas de la emulsión preparada sobre 
agua y sobre aceite, teniendo en cuenta que, para una emulsión o/w, las gotas se dispersan en agua y se aglomeran en aceite.

Por último, se observaron en Microscopio binocular Lancet ${ }^{\circledR}$ Serie XSP las emulsiones preparadas, con aumento de 100x, para observar la homogeneidad de la emulsión y la disposición del sólido en la misma (Hu y col., 2016:300-310).

\section{Obtención de terpineol a partir de emulsión pickering}

Para la obtención de terpineol se utilizó un reactor de vidrio tipo bach provisto de refrigerante a reflujo, montado sobre una manta calefactora con agitación magnética. Se utilizó $1 \mathrm{~g}$ de emulsión aceite:agua y cantidad suficiente de ácido tricloroacético para alcanzar una relación aceite:ácido 1:1. Se llevó a cabo la reacción a $80^{\circ} \mathrm{C}$ con agitación constante durante 8 horas. Al término de las reacciones se separaron por centrifugación las fases orgánica y acuosa del sólido. La fase orgánica se almacenó en frascos color caramelo en refrigerador. El sólido se lavó con alcohol etílico y se secó en estufa a $100^{\circ} \mathrm{C}$ durante 1 hora. Este sólido lavado se reutilizó hasta tres veces en nuevas reacciones. Se calculó la conversión de limoneno en la reacción, la selectividad a-terpineol y el rendimiento. Cada una de las síntesis se realizó por triplicado.

\section{Identificación del producto}

La identificación del terpineol en el producto de reacción se realizó mediante cromatografía de gases acoplada a espectrometría de masas (GC/MS). Se utilizó un equipo Shimadzu modelo QP 5050, con una columna capilar de sílice fundida BP-20 (SGE, Australia), de $25 \mathrm{~m}$ x 0,25 $\mathrm{mm}$ de diámetro interno, recubierta con polietilenglicol $20000(0,25 \mu \mathrm{m}$ de espesor de fase fija). Temperatura en la columna $40^{\circ} \mathrm{C}(8 \mathrm{~min})$, aumentando hasta $180^{\circ} \mathrm{C}\left(3^{\circ} \mathrm{Cmin}^{-1}\right)$, y a $230^{\circ} \mathrm{C}\left(20^{\circ} \mathrm{Cmin}^{-1}\right)$. Temperatura del inyector $250^{\circ} \mathrm{C}$, modo de inyección split, relación de split 1:40; volumen inyectado: $0,2 \mu \mathrm{l}$ de aceite. Fase móvil: helio, 92,6 $\mathrm{kPa}\left(55,9 \mathrm{cmseg}^{-1}\right)$, temperatura en interfase: $250^{\circ} \mathrm{C}$; rango de adquisición de masas: $40-400 \mathrm{mz}^{-1}$. Los patrones de fragmentación de cada componente se compararon con los almacenados en la biblioteca de espectros del software (Allured Publishing Corp, Carol Stream, IL., USA, 2001; Mc Lafferty, y F. Turencek, 4ta ed., University Science Books, Mill Valley, California, 1995.)

\section{Cálculo del rendimiento de reacción}

Se utilizó el método del estándar interno para determinar las concentraciones del limoneno y a-terpineol en el producto de reacción. Se empleó un cromatógrafo Shimadzu GC 14B, con columna polar Megabore DB-WAX P/N 125-7032 de $30 \mathrm{~m}$ de longitud x 0,53 mm de diámetro interno x $1 \mu$ de espesor, conectada a un detector FID. La temperatura del detector fue de $220^{\circ} \mathrm{C}$ y la temperatura de inyector de $180^{\circ} \mathrm{C}$. La temperatura de la columna se mantuvo isotérmica en $30^{\circ} \mathrm{C}$ durante 2 minutos, luego inició una rampa de $5{ }^{\circ} \mathrm{Cmin}-1$ hasta llegar a $200^{\circ} \mathrm{C}$, por último, se mantuvo isotérmica a $200^{\circ} \mathrm{C}$ durante 5 minutos. Como patrón se empleó a-terpineol cromatográfico (Fluka, Sigma-Aldrich) y $(\mathrm{R})(+)$ Limoneno (Fluka, Sigma-Aldrich). Se calculó el rendimiento $(\mathrm{R} \%)$ de la reacción, determinando primeramente la concentración inicial $\left(\mathrm{C}_{\mathrm{i}}\right)$ y final $\left(\mathrm{C}_{\mathrm{f}}\right)$ de limoneno y la concentración final de terpineol $\left(\mathrm{C}_{\text {fTerpineol }}\right)$ en el producto de reacción. Se calculó luego la conversión del limoneno $\left(\mathrm{C}_{\mathrm{v} \text { Limoneno }}\right)$ y la selectividad a terpineol $\left(\mathrm{S}_{\mathrm{vTerpineol}}\right)$ y el rendimiento de la reacción como producto de ambas, ecs. (1)-(3). Para los cálculos se utilizó el promedio de los triplicados realizados. 


$$
\begin{aligned}
& C_{v \text { Limoneno }}=\left(C_{i}-C_{f}\right) / C_{i} \\
& S_{v \text { Terpineol }}=C_{f \text { Terpineol }} / C_{v \text { Limoneno }} \\
& R \%=C_{v \text { Limoneno }} * S_{v \text { Terpineol }}
\end{aligned}
$$

\section{Resultados y discusión}

\section{Caracterización de las emulsiones}

La conductividad de la emulsión aceite:agua desde una relación de 0,2 hasta 1,2 g de agua por gramo de aceite es de $0 \mu \mathrm{S}$, la misma asciende drásticamente a $15 \mu \mathrm{S}$ cuando la masa de agua es de 1,5 por gramo de aceite (Fig. 1), de manera similar a lo que obtuvieron otros autores (Reyes y Di Scipio, 2012:56-69). De acuerdo a este aumento se puede decir que la emulsión sufre una inversión catastrófica (Tadros, 2013:1-75; Piorkowski y Mc Clements, 2014:5-41) por el cambio volumétrico de la fase dispersa, es decir que por encima de 1,5 gramos de agua, la emulsión deja de ser w/o y pasa a ser o/w. Esto se explica porque la fase acuosa contiene algo de electrolito, por lo que la emulsión conduce la electricidad sólo si la fase acuosa es la continua y la oleosa la dispersa (Salager, 2006:185-225). Esto mismo se comprobó con la prueba de la gota, observándose que en relaciones menores a 1,5 gramos de agua por gramo de aceite, las gotas de la emulsión se dispersan en agua, mientras que las de fracciones superiores a este valor se dispersan en aceite. En cambio, al agregar carbón activado a la emulsión, el punto de inversión se da en concentraciones cercanas a 2,0 gramos de agua, aumentando su conductividad de $100 \mu \mathrm{S}$ a $230 \mu \mathrm{S}$, cambiando el tipo de emulsión a o/w (Fig. 2).

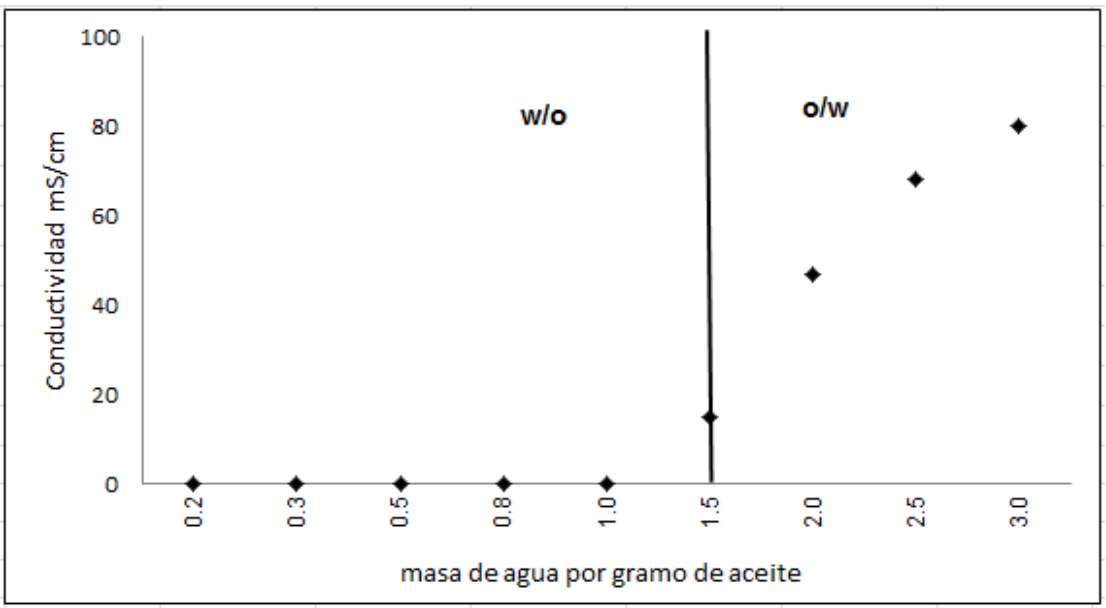

Fig. 1. Conductividad de emulsiones de aceite:agua en función de masa de agua por gramo de aceite. 


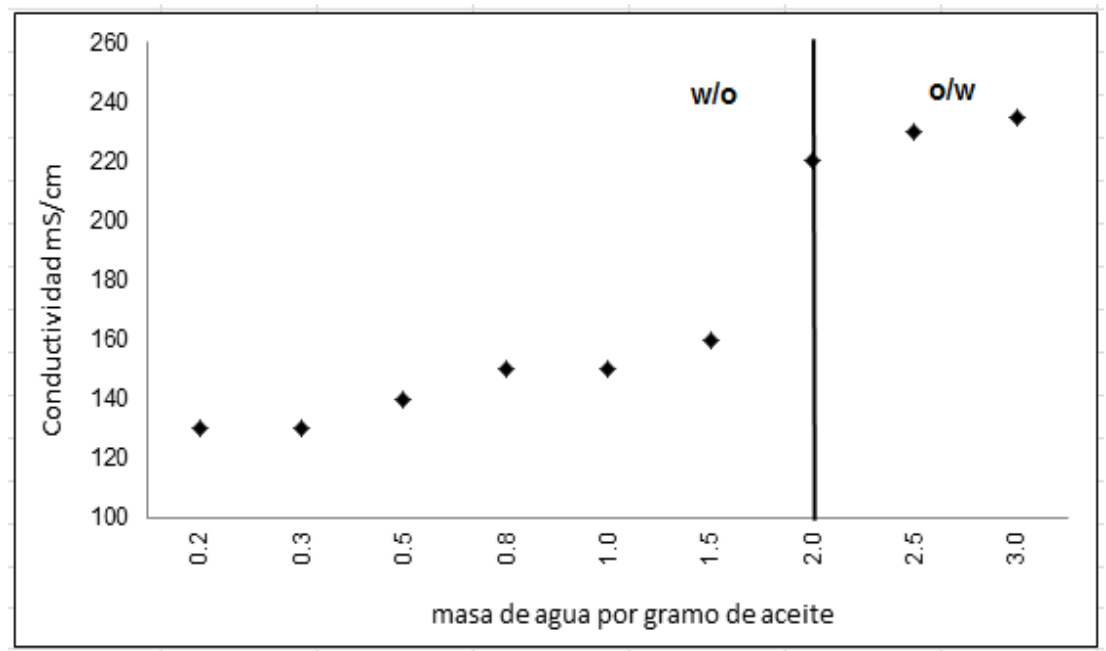

Fig. 2: Conductividad de emulsiones aceite:agua con Carbón activado, en función de masa de agua por gramo de aceite.

La emulsión con bentonita tiene una particularidad: a concentraciones menores de 0,8 gramos de agua, no forma emulsión por lo que no se puede medir su conductividad; además tiene su punto de inversión catastrófica cercano a concentraciones de 2,0 (Fig. 3).

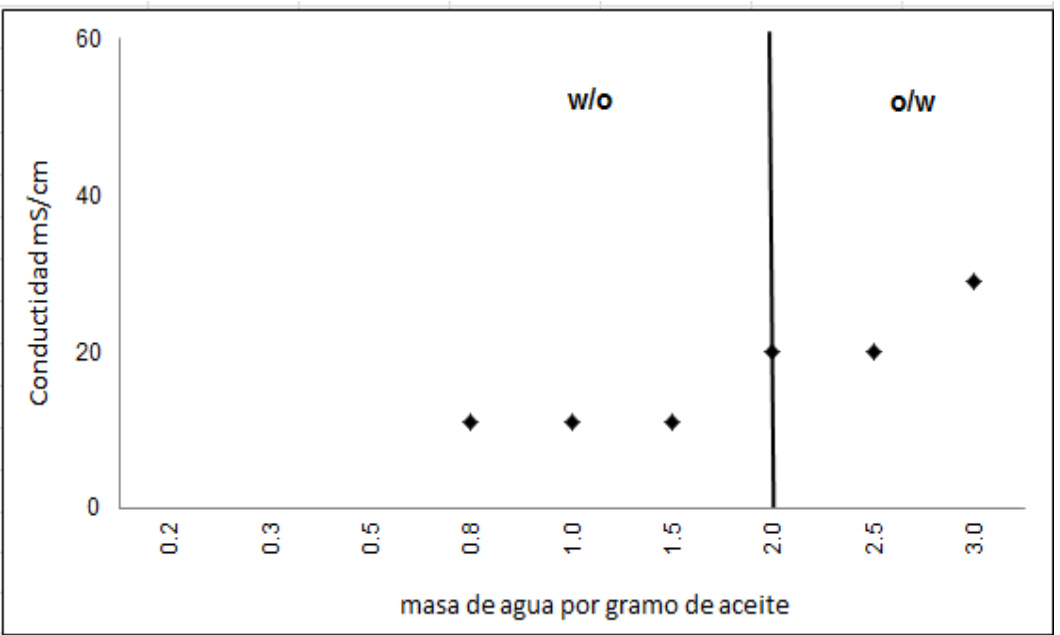

Fig. 3: Conductividad de emulsiones aceite:agua con Bentonita, en función de masa de agua por gramo de aceite.

Las emulsiones con sílica, a concentraciones menores de 0,3 gramos de agua por gramo de aceite forman una emulsión o/w y luego sufre un cambio en su conductividad de $205 \mu \mathrm{S}$ a $130 \mu \mathrm{S}$ evidenciándose con la prueba de la gota que pasa a formarse una emulsión w/o. En concentraciones superiores a 1,0 gramos de agua por gramo de aceite, no forma emulsión (Fig. 4). 


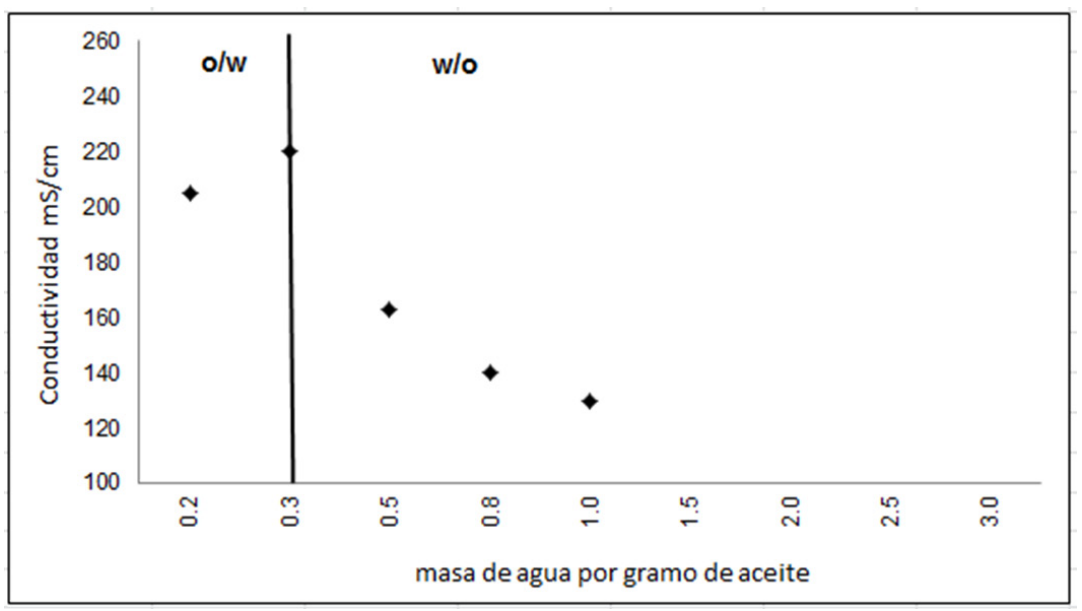

Fig. 4: Conductividad de emulsiones aceite:agua con Sílica, en función de masa de agua por gramo de aceite.

Por último, la emulsión con alúmina sufre el cambio de fase en concentraciones cercanas a 0,3-0,5 gramos de agua por gramo de aceite, cambiando a o/w (Fig. 5).

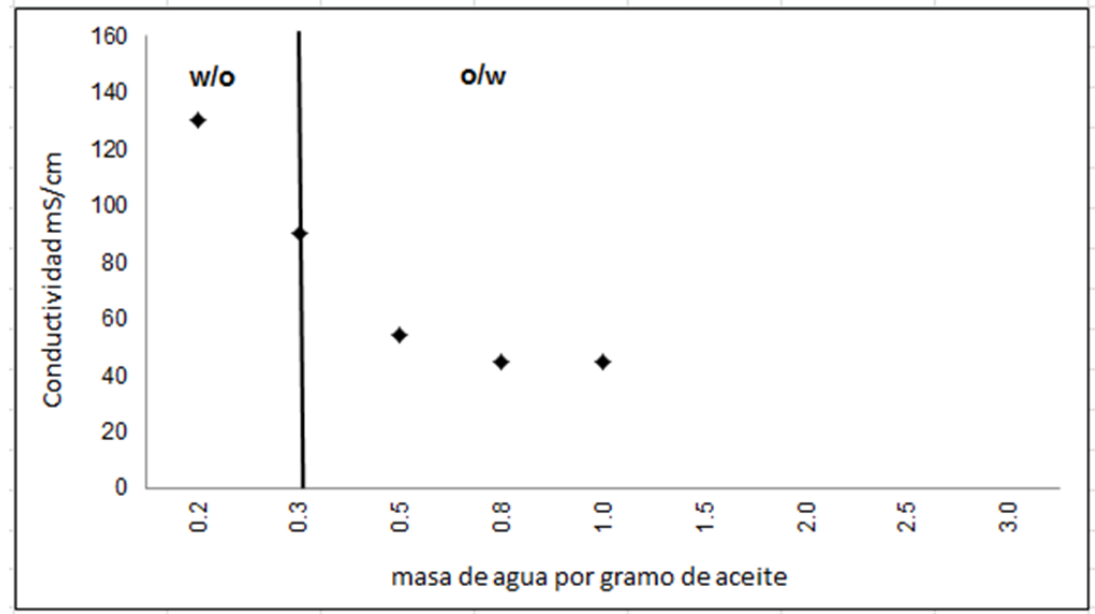

Fig. 5: Conductividad de emulsiones aceite:agua con Alúmina, en función de masa de agua por gramo de aceite.

Estos resultados muestran que la formación de una emulsión pickering estable depende, no solo de la concentración del sólido y de la relación agua:aceite, sino también de la mayor o menor hidrofobicidad del sólido (Hu y col., 2016:300-310; Mirhosseini y col., 2008:47-56). Esto puede observarse en la bentonita y en la sílice que tienen mojabilidad preferente en el aceite a concentraciones superiores de agua, y es por ello que forman emulsiones w/o a diferencias de los demás sólidos que forman emulsiones o/w (Finkle y col., 1923:2780-2788).

De acuerdo a la Tabla 1, las cantidades de sólidos requeridas para formar emulsiones agua:aceite estables de manera tal que no se produzca floculación observable macroscópicamente por el desequilibrio entre fases e interacciones repulsivas (Mirhosseini y col., 2008:47-56) son mayores a los valores informados por Gelot (Gelot y col., 1984:271-303), en 
cambio menores a las reportadas por Carrera (Carrera y col., 2014:447-456).

\begin{tabular}{|l|c|c|}
\hline $\begin{array}{l}\text { Emulsión pickering } \\
\text { con solidos }\end{array}$ & Relación aceite:agua & $\begin{array}{l}\text { Masa de sólido }(\mathrm{g}) \\
\text { por gramo de emulsión }\end{array}$ \\
\hline Carbón activado & $1: 2$ & 0,20 \\
Bentonita & $1: 3$ & 0,11 \\
Alúmina & $2: 1$ & 0,20 \\
Sílica & $2: 1$ & 0,12 \\
\hline
\end{tabular}

Tabla №1 Relaciones de los componentes para obtener una emulsión pickering estable.

Por otra parte, con carbón activado se observa al microscopio muy buena homogeneidad en la emulsión y se pueden ver las zonas amarillentas que corresponden al aceite esencial alrededor del sólido (Fig. 6.a), propias de sólidos hidrofóbicos, similar a lo obtenido por Gelot (Gelot y col., 1984:271-303). En cambio, con bentonita se puede ver que algunas partículas sólidas se encuentran rodeadas de aceite y otras de agua, (Fig. 6.b), esto puede deberse a que la bentonita está compuesta por una mezcla de partículas, tanto hidrófilas como hidrófobas. Otra explicación, puede ser la forma de las partículas, pues para que un sólido penetre en la interfase agua:aceite, ésta debe romperse y parecería ser más probable esta situación con las partículas de carbón, que tienen un mayor grado de curvatura, que con las partículas planas de bentonita (Jameson, 1984: 200-201). Con sílica, (Fig. 6.c) se puede observar una buena emulsión, en concordancia a lo que obtuvo Frelichowska (Frelichowskay col., 2009:7-15) con aceites polares. Además, se ven agregaciones de sólido y aceite formadas por fuerzas de Van der Waals, la cuales evitan la coalescencia y forman un revestimiento rígido alrededor de las gotitas de aceite, comparado a una cáscara de huevo (Tadros, 2013:1-75; Chevalier y Bolzinger, 2013:23-34). También se pueden observar las partículas de alúmina formando una buena emulsión, con aceite dispuesto a su alrededor al igual que con carbón activado (Fig. 6.d).
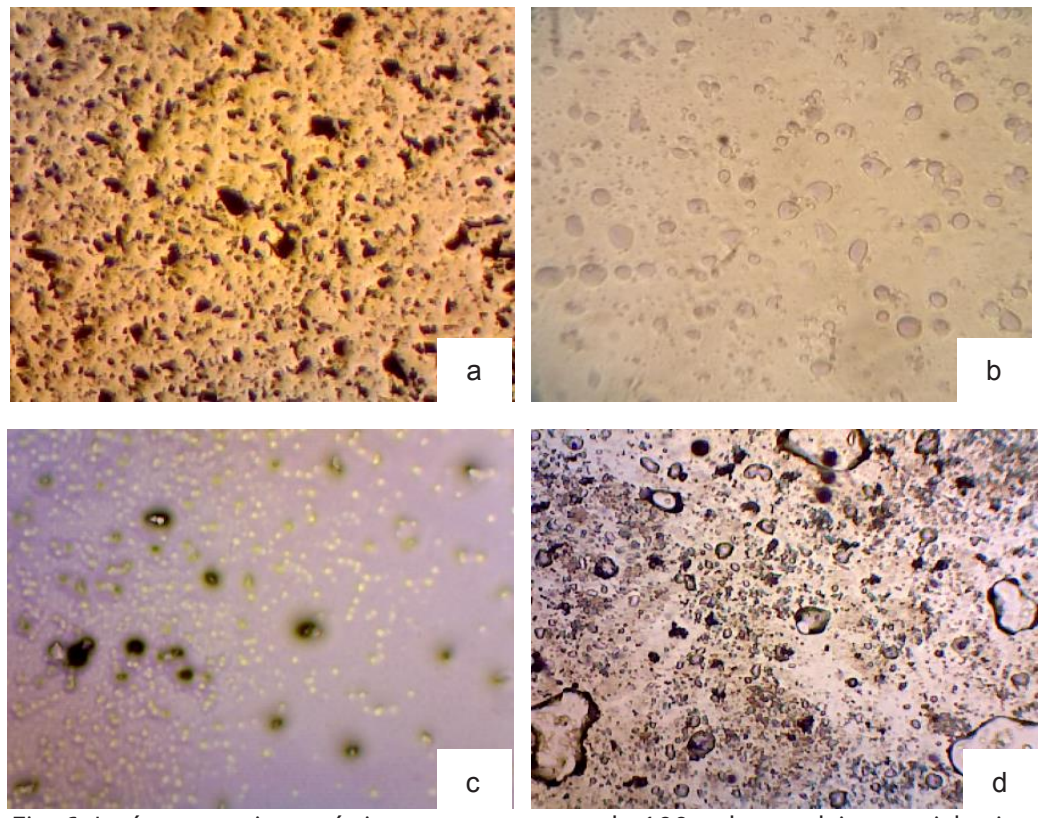

Fig. 6. Imágenes microscópicas con aumento de 100x, de emulsiones pickering de a) Carbón activado, b) Bentonita, c) Sílica y d) Alúmina. 


\section{Obtención de terpineol y rendimiento de la reacción}

Las emulsiones se prepararon de acuerdo a la Tabla 1. Las relaciones de los componentes se seleccionaron de tal manera, que formen una emulsión estable y con la mayor cantidad de agua posible en exceso. Con alúmina y sílica esto no fue posible, ya que no forman emulsión a fracciones mayores de agua. Se comprobó que el agregado de ácido tricloroacético necesario para la reacción y la temperatura de trabajo de $80^{\circ} \mathrm{C}$ no cambian el tipo de emulsión obtenida con esas concentraciones. En cambio, otros autores (Hu y col., 2016:300-316; AfzaliTabar y col., 2017:164-173; Anton y col., 2008:95-99) observaron un cambio de tipo de emulsión al cambiar el $\mathrm{pH}$ de la emulsión.

Se identificó al a-terpineol como producto de reacción y, además, como subproductos se obtuvieron isómeros del $\alpha$-terpineol: $\gamma$, $\beta$ y 4-terpineol como también terpinoleno (isómero del limoneno) con concentraciones relativas menores al $10 \%$.

Los resultados de conversión, selectividad y rendimientos porcentuales en a-terpineol se muestran en la Tabla 2.

\begin{tabular}{|l|c|c|c|c|c|}
\hline \multicolumn{1}{c|}{$\begin{array}{c}\text { Tipo de emulsión } \\
\text { pickering }\end{array}$} & $\mathrm{C}_{\mathrm{v} \text { Limoneno }}(\%)$ & $\mathrm{S}_{\mathrm{v} \text { Terpineol }}(\%)$ & $\mathrm{R} \%$ & $\mathrm{R}_{\mathrm{r} 1} \%$ & $\mathrm{R}_{\mathrm{r} 2} \%$ \\
\hline Carbón activado & $61,6 \pm 5,1$ & $18,0 \pm 3,2$ & $11,0 \pm 2,7$ & $10,8 \pm 1,2$ & $10,5 \pm 2,3$ \\
\hline Bentonita & $57,0 \pm 8,4$ & $32,0 \pm 4,2$ & $18,0 \pm 0,3$ & $17,7 \pm 1,1$ & $17,0 \pm 1,2$ \\
\hline Sílica & $80,0 \pm 9,1$ & $54,0 \pm 8,5$ & $43,0 \pm 7,3$ & $42,3 \pm 3,5$ & $42,1 \pm 3,8$ \\
\hline Alúmina & $50,0 \pm 3,2$ & $30,0 \pm 0,8$ & $15,0 \pm 0,8$ & $15,5 \pm 0,9$ & $14,9 \pm 0,6$ \\
\hline Sin sólido & $35,0 \pm 2,5$ & $20,0 \pm 2,8$ & $7,0 \pm 1,7$ & -- & -- \\
\hline
\end{tabular}

Tabla 2: Conversiones de limoneno $\left(\mathrm{C}_{\mathrm{v} \text { Limoneno }}\right)$, Selectividad a terpineol $\left(\mathrm{S}_{\mathrm{v} \text { Terpineol }}\right)$, Rendimiento de la reacción $(\mathrm{R} \%)$ Rendimiento con primer Recupero $\left(R_{r 1} \%\right)$, Rendimiento con segundo Recupero $\left(R_{r 2} \%\right)$ con emulsiones pickering.

La mayor conversión (80\%) se obtuvo usando sílica con una selectividad del 54\% a terpineol, bastante más alta que otros autores: conversión del $54 \%$ con selectividad del $45 \%$ con catalizador de zeolitas (Yadav y col., 2009:101-109), selectividad del 5,9\% con heteropoliácidos como catalizador, con altos porcentajes de isómeros como subproductos (Avila y col., 2008:1460-1462). Otros estudios demostraron una conversión y una selectividad del $30 \%$ y $45 \%$ respectivamente en 3 horas y $83 \%$ y 32\% respectivamente en 24 horas (RoblesDutenhefner y col., 2001:33-42). Además, todos los rendimientos obtenidos con emulsión pickering fueron más altos que los alcanzados sin partículas sólidas en el medio de reacción. Se logró también reutilizar los sólidos recuperados hasta dos veces consecutivas sin observarse modificaciones en el rendimiento de la reacción ( $\mathrm{Rr} 1 \%$ y Rr2\%), cuyos valores se observan en la Tabla 2.

Es importante aclarar que en estas reacciones se utilizan sólidos como partícula sólida emulsionante, aumentando la superficie de contacto entre las fases agua:aceite, no poseen centros activos, y por lo tanto su precio no es elevado. Otros autores han llevado a cabo esta reacción, pero con distintos catalizadores como zeolitas beta con iones de metales de transición (Yadav y col., 2009:101-109), ácido fosfotúngstico y molibdénico soportado en titania y sílica (Avila y col., 2008:1460-1462), sílica soportada por heteropoliácidos H3PW12O40 (Robles-Dutenhefner y col., 2001:33-42), siendo éstos de alto precio o con metodologías de obtención complejas, comparado con los sólidos utilizados en este trabajo. 


\section{Conclusiones}

A través del uso de emulsiones pickering se logró aumentar la superficie de contacto entre dos reactivos y de esta manera aumentar considerablemente el rendimiento en la reacción. Con sílica se obtienen mayores rendimientos a a-terpineol, esto puede ser porque este sólido forma una emulsión muy estable con partículas de tamaño y formas homogéneas.

Este método es novedoso ya que se obtienen altas conversiones y rendimientos, sin el uso de catalizadores cuya asequibilidad muchas veces es difícil por su alto precio y su técnica compleja de obtención y representa un nuevo uso para este tipo de emulsiones que va adquiriendo innumerables y exitosas aplicaciones en distintos campos. 


\section{Referencias}

AfzaliTabar, M., Alaeib, M., Ranjineh Khojasteha, R., Motieea, F., Rashidi, A. M. (2017). "Preference of multi-walled carbon nanotube (MWCNT) to single-walled carbon nanotube (SWCNT) and activated carbon for preparing silica nano hybrid pickering emulsion for chemical enhanced oil recovery (C-EOR)”. Journal of Solid State Chemistry, 245, 164-173. http://dx.doi.org/10.1016/j.jssc.2016.10.017

Anton, N., Benoit1, J. P., Saulnier, P. (2008). "Particular conductive behaviors of emulsion phase inverting”. Journal of Drug Delivery Science and Technology, 18 (2), 95-99. https:// doi.org/10.1016/S1773-2247(08)50015-3

Aranberri, I. (2009). "Estabilidad de las emulsiones de Pickering utilizadas en la elaboración de productos agroquímicos”. Anales de la Real Sociedad Española de Química, 105 (1), 13-17. Disponible en: https://dialnet.unirioja.es/servlet/ articulo? codigo=2931241

Aranberri, I., Binks, B.P., Clint, J.H. y Fletcher, P.D.I. (2006). “Elaboración y caracterización de emulsiones estabilizadas por polímeros y agentes tensioactivos". Revista Iberoamericana de Polímeros, 7 (3), 211-231. Disponible en: http://www.ehu.eus/ reviberpol/pdf/AGO06/aranberri.pdf

Aveyard, R., Binks, B. P., Clint, J. H. (2003). "Emulsions stabilised solely by colloidal particles”. Advances in Colloid and Interface Science, 100, 503-546. https://doi.org/10.1016/ $\underline{\text { S0001-8686(02)00069-6 }}$

Avila, M., Comelli, N., Firpo, N., Ponzi, E. y Ponzi, M. (2008). "Hydration and acetylation of limonene.Supported heteropolyacids". Journal of the Chilean Chemical Society, 53, 14601462. https://doi.org/10.4067/S0717-97072008000100027

Baptistella, L. H. B., Imamura, P. M., Melo, L. V. de, \& Castello, C. (2009). "Preparação do (+)-t-terpineol a partir do (+)-limoneno: monoterpenos de odor agradável em um projeto para química orgânica experimental”. Química Nova, 32 (4), 1069-1071. https://doi. org/10.1590/s0100-40422009000400042

Bathia, S., Mc Ginty, D., Foxenberg, R., Letizia, C. y Api A. (2008). "Fragrance material review on terpineol”. Food and Chemical Toxicology,46, S275-S279. https://doi. org/10.1016/j.fct.2008.06.075

Cáceres, L.M., Zambón, S.N., Velasco, G.A., Chamorro, E.R. (2018). “Efecto del Solvente en la Reacción de Obtención de a-Terpineol a partir de Aceite de Pomelo”. Revista Tecnología y Ciencia de la Universidad Tecnológica Nacional, 31, 30-37. http://rtyc.utn.edu.ar/index. $\mathrm{php} / \mathrm{rtyc} / \mathrm{article} / \mathrm{view} / 97$

Carrera, Y., García-Marquez, E., Aguirre-Mandujano, E., Rodríguez-Huezo, M. E., y AlvarezRamirez, J. (2014). "Stabilizing oil-in.water emulsions with em bean (Pachyrhizus erosus L. Urban) solids”. Revista Mexicana de Ingeniería Química, 13 (2), 447-456. Disponible en: http://www.scielo.org.mx/pdf/rmiq/v13n2/v13n2a10.pdf

Castellanos Molina, F. R. (2007). "Biotransformación de limoneno, a-pineno y aceites esenciales de Naranja y mandarina empleando Aspergilius niger”. Tesis (Maestría en Química), Universidad Industrial De Santander, Colombia, 80-82.

Chevalier, Y., Bolzinger, M. A. (2013). "Emulsions stabilized with solid nanoparticles: 
Pickering emulsions". Colloids and Surfaces A: Physicochemical and Engineering Aspects, 439, 23-34. https://doi.org/10.1016/j.colsurfa.2013.02.054

De Campo, L., Yaghmur, A., Garti, N., Leser, M. E., Folmer, B. y Glatter, O. (2004). "Fivecomponent food-grade microemulsions: structural characterization by SANS".Journal of Colloid and Interface Science, 274, 251-267. https://doi.org/ 10.1016/j.jcis.2004.02.027

Finkle, P., Draprx, H. D. y Hildgdra, J. H. (1923). “The theory of emulsification1”. Journal of American Chemical Society, 45 (12), 2780-2788. https://doi.org/10.1021/ ja01665a002

Frelichowska, J., Bolzinger, M. A., Valour, J. P. (2009). "Pickering w/o emulsions: Drug release and topical delivery". International Journal of Pharmaceutics, 368 (1-2), 7-15. https://doi.org/10.1016/j.ijpharm.2008.09.057

Garti, N. (2003). "Microemulsions as microreactors for food applications". Current Opinion in Colloid and Interface Science,8 (2), 197-211. https://doi.org/10.1016/ S13590294Ž03.00022-0

Gelot, A., Friesen, W. y Hamza, H.A. (1984). "Emulsification of oil and water in the presence of finely divided solids and surface-actjve agents”. Colloids and Surfaces,12, 271303. https://doi.org/10.1016/0166-6622(84)80105-5

Gers-Barlag, H. y Müller, A., "Sistemas de dispersión fina, sin emulsionantes, de tipo aceite en agua o agua en aceite”, Pat. Num. ES2276495T3, Oficina Española de patentes y marcas, España, Mar. 22, (2000). Disponible en: http://digital.csic.es/ bitstream/10261/6886/1/2276916_T3.pdf

Gusevskaya, E. V. (2014). "Reactions of Terpenes Catalyzed by Heteropoly Compounds: Valorization of Biorenewables". ChemCatChem, 6 (6), 1506-1515. https://doi.org/10.1002/ cctc. 201400052

Hu, Y., Yin, S., Zhu, J., Qi, J., Guo, J., Wu, L., Tang, C. y Yang, X. (2016). "Fabrication and characterization of novel Pickering emulsions and Pickering high internal emulsions stabilized by gliadin colloidal particles”. Food Hydrocolloids, 61, 300-310. https://doi. org/10.1016/j.foodhyd.2016.05.028

Jameson, G. (1984). Experimental Techniques in flotation. En: The scientific Basis of flotation, (I. Kennet). Paises Bajos: Martinus Nijhoff Publishers, 200-201.

Kasaka, Y., Bibouche, B., Volovych, I., Schwarze, M. y Schomäcker, R. (2016). "Investigation of phase behaviour of selected chemical reaction mixtures in microemulsions for technical applications". Colloids and Surfaces A: Physicochemical Engineering Aspects, 494, 49-58. https://doi.org/10.1016/j.colsurfa.2016.01.013

Laguna, O. H., Centeno, M. A., Boutonnet, M. J. y Odriozola, A. (2016). "Au-supported on Fe-doped ceria solids prepared in water-in-oil microemulsions: Catalysts for CO oxidation". Catalysis Today, 278, 140-149. https://doi.org/10.1016/j.cattod.2016.05.059

Low, L. E., Tey, B.T., Ong, B.H., Chan, E.S., Tang, S.Y. (2017). "Palm olein-in-water Pickering emulsion stabilized by Fe3O4-cellulose nanocrystal nanocomposites and their responses to pH”. Carbohydrate Polymers,155, 391-399. https://doi.org/10.1016/j.carbpol.2016.08.091

Maróstica, M. y Pastore, G. (2007). "Production of R-(+)-a-terpineol by the biotransformation of limonene from orange essential oil, using cassava waste water as 
medium”. Food Chemistry, 101, 345-350. https://doi.org/10.1016/j.foodchem.2005.12.056

Mirhosseini, H., Tan, C. P., Hamid, N. S. A. y Yusof, S. (2008). "Effect of Arabic gum, xanthan gum and orange oil contents on $\zeta$-potential, conductivity, stability, size index and $\mathrm{pH}$ of orange beverage emulsion". Colloids and Surfaces A:

PhysicochemicalEngineeringAspects, 315, 47-56. http://dx.doi.org/10.1016/ j.colsurfa.2007.07.007

Monfort, T. (2004). Salsas. En: Química y bioquímica de los alimentos II. (J. Boatella Riera, R. Codoni Salcedo, P. López Alegret) España: Publicaciones i Edicions de la Universitate de Barcelona, 143-148. ISBN 84-475-2836-7

Newton, A. E., Fairbanks, A. J., Golding, M., Andrewes, P. y Gerrard, J. A. (2015).“The influence of emulsion structure on the Maillard reaction of ghee”. Food Chemistry, 173, 1243-1249. https://doi.org/10.1016/j.foodchem.2014.10.147

Oyedemi, S., Okoh, A., Mabinya, L., Pirochenva, G. y Afolayan, A. (2009). “The proposed mechanism of bactericidal action of eugenol, a-terpineol and $\gamma$-terpinene against Listeria monocytogenes, Streptococcus pyogenes, Proteus vulgaris and Escherichia coli”.The African Journal of Biotechnology, 8, 1280-1286. https://doi.org/10.4314/ ajb.v8i7.60106

Pereira, T. C., Conceição, C. A. F., Khan, A., Fernandes, R. M. T., Ferreira, M. S., Marques, E. P. y Marques, A. L. B. (2016). "Application of electrochemical impedance spectroscopy: A phase behavior study of babassu biodiesel-based microemulsions". Spectrochimica Acta Part A: Molecular and Biomolecular Spectroscopy, 168, 60-64. https://doi.org/10.1016/j. $\underline{\text { saa.2016.05.034 }}$

Piorkowski, D. T. y McClements, D. J. (2014). "Beverage emulsions: Recent developments in formulation, production, and applications". Food Hydrocolloids, 42, 5-41. https://doi. org/10.1016/j.foodhyd.2013.07.009

Piriyaprasarth, S., Maneerat, J. y Pornsak, S. (2016). "Stability of rice bran oil-in-water emulsions stabilized by pectinezein complexes: Effect of composition and order of mixing”. Food Hydrocolloids, 61, 589-598. https://doi.org/10.1016/ j.foodhyd.2016.06.015

Popp, N., Kutuzov, S. y Böker, A. (2010). "Various Aspects of the interfacial self- Assembly of nanoparticles”. Advanced Polymery Science, 228, 39-58. https://doi.org/10.1007/12_2010_52

Ramírez Galilea, A. (2012). "Determinación de coeficientes de transferencia de materia en emulsiones estabilizadas por nanohíbridos tipo NTC/Soporte”. Tesis (Ingeniería Química y Tecnología del medio ambiente). Escuela de Ingeniería y Arquitectura, Zaragoza, 8-12.

Reyes, P. y Di Scipio, S. (2012). "Caracterización físico-química de emulsiones de aceite de maíz en agua”. Revista de la Facultad de Ingeniería Universidad Central de Venezuela, 27 (1), 56-69. Disponible en: https://www.researchgate.net/ publication/262548935_

Caracterizacion_fisico-quimica_de_emulsiones_de_aceite_de_maiz_en_agua

Robles-Dutenhefner, P., Da Silva, K., Siddiqui, M., Kozhevnikov, I. y Gusevskaya, E. (2001). "Hydration and acetoxylation of monoterpenes catalyzed by heteropoly acid". Journal of Molecular Catalysis A: Chemical, 175, 33-42.https://doi.org/10.1016/ S1381-1169(01)00217-5

Rottava, I., Toniazzo, G., Cortina, P. F., Martello, E., Grando, C. E., Lerin, L. A., Treichel, H, Mossi, A., Oliveira, D., Cansian, R. L., Antunes, O.A.C. y Oestreicher, E. G. (2010). "Screening of microorganisms for bioconversion of $(-) \beta$-pinene and $\mathrm{R}-(+)$-limonene 
to a-terpineol. LWT - Food Science and Technology, 43 (7), 1128-1131. https://doi. org/10.1016/j.lwt.2010.03.001

Salager, J. L. (2006). Emulsion Phase Inversion Phenomena. En: Emulsions and emulsion stability, (A. T. Hubbard, ed.). Florida: Taylor \& Francis Group, 185-225

Salager, J. L., Loaiza-Maldonado, I., Miñana-Pérez, M. y Silva, F. (1982). "Surfactantoil-water systems near the affinity inversionpart: relationship between equilibrium phasebehavior and emulsion type and stability". Journal of Dispersion Science and Technology, 3, 279-292. https://doi.org/10.1080/01932698208943642

Salager, J.L., López-Castellanos, G., Miñana-Perez, N., Parra, C., Cucuphat, C. Graciaa, A. y Lachaise, J. (1991). "Surfactant-oil-water systems near the affinity inversion: Part VII: Phase behavior and emulsions with polar oils”. Journal of Dispersion Science and Technology, 12, 59-67. https://doi.org/10.1080/01932699108913105

Salamanca, M., Licea, Y., Echavarría, A., Faro, A. y Palacio, L. (2009). “Uso de materiales laminares tipo cobalto-molibdato como potenciales catalizadores en la transformación de limoneno". Avances en Química, 4, 93-100. Disponible en: https://www.redalyc.org/ pdf/933/93313210001.pdf

Schramm, L. (2005). Emulsions, Foams, and Suspensions: Fundamentals and Applications. Editorial Wiley-VCH, Alemania, 10-12.

Starks, C. M. (1971). "Phase-transfer catalysis. I. Heterogeneous reactions involving anion transfer by quaternary ammonium and phosphonium salts". Journal of the American Chemical Society, 93 (1), 195-199. https://doi.org/10.1021/ja00730a033

Tadros, T. F. (2013). Emulsion Formation, Stability, and Rheology. En Emulsion Formation and Stability (T. F. Tadros, ed.). Alemania: Wiley-VCH Verlag GmbH \& Co, 1-75.

Tan, Q., Day, D. y Cadwallader, K. (1998). "Bioconversion of (R)-(+)-limonene by P digitatum”. Process Biochemistry, 33 (1), 29-31. https://doi.org/10.1016/S0032-9592(97)000484

Varka, E. M., Ampatzidis, C., Kostoglou, M., Karapantsios, T. y Dutschk, V. (2010). "On the use of electrical conductance measurements for the stability of oil-in-water Pickering emulsions". Colloids and Surfaces A: Physicochemical and Engineering Aspects, 365, 181188. https://doi.org/10.1016/j.colsurfa.2010.02.017

Vasek, O., Cáceres, L. M., Chamorro, E., y Velasco, G. (2015). "Antibacterial activity of citrus paradisi essential oil”.Journal of Natural Products, 8, 16-26. Disponible en: http:// journalofnaturalproducts.com/Volume8/5_Res_paper-4.pdf

Welsh, F., Murray, W. y Williams, R. (1989).“Microbiological and enzymatic production of flavour and fragance chemicals”. Critical Reviews in Biotechnology, 9, 105-169. https://doi. org/10.3109/07388558909040617

Wen, C., Yuan, Q., Liang, H., \& Vriesekoop, F. (2014). "Preparation and stabilization of d-limonene Pickering emulsions by cellulose nanocrystals”. Carbohydrate Polymers, 112, 695-700. https://doi.org/10.1016/j.carbpol.2014.06.051

Xing, Q., Song, J., You, X., Xu, D., Wang, K., Song, J., Guo, Q., Li, P., Wu, C. y Hu, H. (2016). "Microemulsions containing long-chain oil ethyl oleate improve the oral bioavailability 
of piroxicam by increasing drug solubility and lymphatic transportation simultaneously". International Journal of Pharmaceutics, 511 (2), 709-718. https://doi.org/10.1016/j. ijpharm.2016.07.061

Yadav, M., Patil, M., y Jasra, R. (2009). "Acetoxylation and hydration of limonene and pinene using cation exchanged zeolite beta". Journal of Molecular Catalysis A: Chemical, 297 (2), 101-109. https://doi.org/10.1016/j.molcata.2008.09.017

Yaghmur, A., Aserin, A., Abbas, A. y Garti, N. (2005). "Reactivity of furfural-cysteine model reaction in food-grade five-component nonionic $\mathrm{O} / \mathrm{W}$ microemulsions. Colloids and Surfaces A: Physicochem”. Engineering Aspects, 253, 223-234. https://doi.org/10.1016/j. colsurfa.2004.10.114

Yang, H., Zhang, H., Peng, J., Zhang, Y., Du, G. y Fang, Y. (2017). "Smart magnetic ionic liquid-based Pickering emulsions stabilized by amphiphilic Fe3O4 nanoparticles: Highly efficient extraction systems for water purification". Journal of Colloid and Interface Science, 485, 213-222. https://doi.org/10.1016/j.jcis.2016.09.023

Zhang, K., Wu, W., Guo, K., Chen, J.F. y Zhang, P.Y. (2009). "Magnetic polymer enhanced hybrid capsules prepared from a novel Pickering emulsion polymerization and their application in controlled drug release". Colloids and Surfaces A: Physico chemical Engineering Aspects, 349, 110-116. https://doi.org/10.1016/j.colsurfa.2009.08.005 